\title{
Linear Complexity of Generalized Cyclotomic Sequences of Order 4 over $\mathbb{F}_{l}$
}

\author{
Yuhua Sun, ${ }^{1}$ Qiuyan Wang $\mathbb{D D}^{2,3}$ Yang Yan ${ }^{D},{ }^{4}$ Tongjiang Yan, ${ }^{1}$ and $\mathrm{Hui}^{\mathrm{Li}}{ }^{5}$ \\ ${ }^{1}$ College of Science, China University of Petroleum Qingdao, Shandong 266580, China \\ ${ }^{2}$ School of Computer Science and Technology, Tiangong University, Tianjin 300387, China \\ ${ }^{3}$ Provincial Key Laboratory of Applied Mathematics, Putian University, Putian, Fujian, China \\ ${ }^{4}$ School of Information Technology and Engineering, Tianjin University of Technology and Education, Tianjin 300222, China \\ ${ }^{5}$ State Key Laboratory of Integrated Service Networks, School of Cyber Engineering, Xidian University, Xi'an 710071, \\ Shanxi, China
}

Correspondence should be addressed to Qiuyan Wang; wangyan198801@163.com

Received 12 July 2019; Accepted 17 December 2019; Published 11 March 2020

Academic Editor: Massimiliano Zanin

Copyright (c) 2020 Yuhua Sun et al. This is an open access article distributed under the Creative Commons Attribution License, which permits unrestricted use, distribution, and reproduction in any medium, provided the original work is properly cited.

Generalized cyclotomic sequences of period $p q$ have several desirable randomness properties if the two primes $p$ and $q$ are chosen properly. In particular, Ding deduced the exact formulas for the autocorrelation and the linear complexity of these sequences of order 2 . In this paper, we consider the generalized sequences of order 4 . Under certain conditions, the linear complexity of these sequences is developed over a finite field $\mathbb{F}_{l}$. The results show that, in many cases, they have high linear complexity.

\section{Introduction}

Let $l$ be a prime number and $\mathbb{F}_{l}$ denote a finite field with $l$ elements. A sequence $\lambda^{\infty}=\lambda_{0} \lambda_{1} \cdots \lambda_{n-1} \cdots$ is called to be $n$ periodic if $\lambda_{i}=\lambda_{i+n}$ for all $i \geq 0$. Periodic sequences with certain properties are widely used in software testing, radar systems, stream ciphers, and so on. For cryptography applications, the linear complexity is an important factor. It is defined to be the length of the shortest linear feedback shift register which generates this sequence. For cyclotomic sequences, many researchers are devoted to studying their random properties [1-8]. The generalized cyclotomic sequences have been described and studied $[9-11,12,13]$ for the past decades. A number of periodic sequences with attractive random properties have been constructed $[6,14-20]$.

Let $p$ and $q$ be two distinct odd primes with $\operatorname{gcd}(p-1, q-1)=d$. Define $n=p q$ and $e=(p-1)$ $(q-1) / d$. The Chinese reminder theorem guarantees that there exists a common primitive root $g$ of both $p$ and $q$. Let $x$ be an integer satisfying

$$
\begin{aligned}
& x \equiv g(\bmod p), \\
& x \equiv 1(\bmod q) .
\end{aligned}
$$

Whiteman proved that [21]

$$
\mathbb{Z}_{n}^{*}=\left\{g^{i} x^{j}: i=0,1, \ldots, e-1 ; j=0,1, \ldots, d-1\right\},
$$

where $\mathbb{Z}_{n}^{*}$ denotes the set of all invertible elements of the residue class ring $\mathbb{Z}_{n}$.

The generalized cyclotomic classes $D_{j}(0 \leq j \leq d-1)$ of order $d$ with respect to $n$ are defined by [21]

$$
D_{j}=\left\{g^{i} x^{j}: i=0,1, \ldots, e-1\right\}, \quad 0 \leq j \leq d-1,
$$

where the multiplication is that of $\mathbb{Z}_{n}$. Clearly, the cosets $D_{j}$ depend on the choice of the common primitive root $g$ if $d \geq 3$. It is not hard to prove that [21]

$$
\mathbb{Z}_{n}^{*}=\bigcup_{j=0}^{d-1} D_{j}, D_{i} \cap D_{j}=\varnothing, \quad \text { for } i \neq j,
$$

where $\varnothing$ denotes the empty set. Define 


$$
\begin{aligned}
& P=\{p, 2 p, \ldots,(q-1) p\}=p \mathbb{Z}_{q}^{*} \\
& Q=\{q, 2 q, \ldots,(p-1) q\}=q \mathbb{Z}_{p}^{*} .
\end{aligned}
$$

Then,

$$
\mathbb{Z}_{n}=\bigcup_{i=0}^{d-1} D_{i} \cup\{0\} \cup P \cup Q
$$

Let $S$ be a nonempty subset of $\{0,1, \ldots, d-1\}$ and

$$
\sum_{S}=\bigcup_{i \in S} D_{i}
$$

We define the binary sequence $\lambda^{\infty}=\lambda_{0} \lambda_{1} \cdots \lambda_{n-1} \cdots$ of period $n$ as follows:

$$
\lambda_{i}= \begin{cases}1, & \text { if } i \bmod n \in\left(P \cup \Sigma_{S}\right), \\ \rho, & \text { if } i \bmod n \in\{0\} \\ 0, & \text { otherwise, }\end{cases}
$$

where $i \geq 0$ and $\rho \in\{0,1\}$. For $\rho=0$ and $S=\{0\}$, the linear complexity of these sequences over $\mathbb{F}_{2}$ has been calculated by Ding [16] with $d=2$ and $\mathrm{Hu}$ et al. [20] with $d=4$. Furthermore, for $S=\{0,2, \ldots, d-2\}$ and $\rho=0$, Ding [9] determined the linear complexity of the two-prime sequences over a finite field $\mathbb{F}_{l}$ and used these sequences to construct several classes of cyclic codes over $\mathbb{F}_{l}$ with optimal or almost optimal property, where $\operatorname{gcd}(l, n)=1$. In this paper, we only consider the case $\rho=1, d=4$, and $S=\{0,1\}$. Under the assumption that $(n-1) / 4 \equiv 0(\bmod l)$ or $l \notin D_{0}$, we calculate the linear complexity of these sequences over the finite field $\mathbb{F}_{l}$. The results show that, in many cases, these sequences have high linear complexity.

This paper is organized as follows. Section 2 presents basic notations and results of periodic sequences and the generalized cyclotomy [21]. In Section 3, we give an expression for the linear complexity of the generalized cyclotomic sequences over $\mathbb{F}_{l}$. In the last section, we present concluding remarks of this paper.

\section{Preliminaries}

Firstly, we give the definition and formula of linear complexity of periodic sequences over a finite field. See [22] or [23] for more details.

Let $l$ be a prime number and $\lambda^{\infty}=\lambda_{0} \lambda_{1} \cdots \lambda_{n-1} \cdots$ be a periodic sequence over $\mathbb{F}_{l}$ with period $n$, where $\lambda_{i} \in \mathbb{F}_{l}$ for $i \geq 0$. The sequence $\lambda^{\infty}$ can be viewed as a power series

$$
\begin{aligned}
\Lambda^{\infty}(x) & =\sum_{i=0}^{\infty} \lambda_{i} x^{i}=\frac{\Lambda(x)}{1-x^{n}}, \\
\Lambda(x) & =\lambda_{0}+\lambda_{1} x+\cdots+\lambda_{n-1} x^{n-1} \in \mathbb{F}_{l}[x],
\end{aligned}
$$

in the power series ring $\mathbb{F}_{l}[[x]]$.

Let $h(x)=\operatorname{gcd}\left(\Lambda(x), 1-x^{n}\right)$, then

$$
\begin{aligned}
\Lambda^{\infty}(x) & =\frac{w(x)}{v(x)}, \\
v(x) & =\frac{1-x^{n}}{h(x)}, \\
w(x) & =\frac{\Lambda(x)}{h(x)},
\end{aligned}
$$

where $w(x), v(x), h(x) \in \mathbb{F}_{l}[x]$.

Definition 1. The polynomial $v(x)$ is called the minimal polynomial of the periodic sequence $\lambda^{\infty}$ over $\mathbb{F}_{l}$. The $\operatorname{deg} v(x)=n-\operatorname{deg} h(x)$ is called the linear complexity of the sequence $\lambda^{\infty}$ over $\mathbb{F}_{l}$, which is denoted by $L_{l}(\lambda)$.

Indeed, $L_{l}(\lambda)$ is the length of the shortest linear feedback shift register which generates the sequence $\lambda^{\infty}$.

If $\operatorname{gcd}(n, l)=1$, then $1-x^{n}$ has $n$ distinct roots $\zeta_{n}^{i}(0 \leq i \leq n-1)$ in the algebraic closure $\Omega_{l}$ of $\mathbb{F}_{l}$, where $\zeta_{n}$ denotes the $n$-th primitive root of unity. It is easy to see that

$$
L_{l}(\lambda)=n-\left|\left\{i: 0 \leq i \leq n-1, \Lambda\left(\zeta_{n}^{i}\right)=0\right\}\right| .
$$

In order to determine the linear complexity of generalized cyclotomic sequences, we introduce generalized cyclotomy.

Let symbols be the same as in the introduction and $d=\operatorname{gcd}(p-1, q-1)=4$. The generalized cyclotomic numbers of order 4 with respect to $n$ is defined by

$$
(i, j)=\left|\left(D_{i}+1\right) \cap D_{j}\right|, \quad \text { for } 0 \leq i, j \leq 3 .
$$

By the well-known theorem ([24], P.128), there are exactly two representations of $n$ in the form $n=a^{2}+4 b^{2}$ with $a \equiv 1(\bmod 4)$ and the sign of $b$ indeterminate.

Let $g_{1}$ and $g_{2}$ be a fixed primitive root of $p$ and $q$, respectively. For $i=1,2$, let $x_{i}$ and $y_{i}$ be the integers given uniquely by

$$
\begin{aligned}
p & =x_{1}^{2}+4 y_{1}^{2}, \\
q & =x_{2}^{2}+4 y_{2}^{2}, \\
x_{1} & \equiv x_{2} \equiv 1(\bmod 4), \\
2 y_{1} & \equiv-\left(-g_{1}\right)^{(p-1) / 4} x_{1}(\bmod p), \\
2 y_{2} & \equiv-\left(-g_{2}\right)^{(q-1) / 4} x_{2}(\bmod q) .
\end{aligned}
$$

Define $a$ and $b$ to be integers satisfying

$$
\begin{aligned}
& a=x_{1} x_{2}+4\left(\frac{2}{p}\right)\left(\frac{2}{q}\right) y_{1} y_{2}, \\
& b=x_{2} y_{1}-\left(\frac{2}{p}\right)\left(\frac{2}{q}\right) x_{1} y_{2},
\end{aligned}
$$

where $(\div)$ denotes the Legendre symbol.

It is clear that $a \equiv 1(\bmod 4)$ and $n=a^{2}+4 b^{2}$ is one of the two representations of $n$. The following lemma shows that the generalized cyclotomic numbers of order 4 with respect to $n$ depend uniquely on this representation. 
Lemma 1 (see [19], Theorem IV.1.). Let $p \equiv q \equiv 1(\bmod 4)$ be two distinct primes with the fixed primitive roots $g_{1}$ and $g_{2}$, respectively. $M=((p-2)(q-2)-1) / 4$, and $a$ and $b$ are the integers defined in (14).

If $p \neq \equiv q(\bmod 8)$, then in Table $1,8 A=-a+2 M+3$, $8 B=-a-4 b+2 M-1,8 C=3 a+2 M-1,8 D=-a+4 b+$ $2 M-1$, and $8 E=a+2 M+1$.

If $p \equiv q(\bmod 8)$, then in Table $2,8 A=3 a+2 M+5$, $8 B=-a+4 b+2 M+1,8 C=-a+2 M+1,8 D=-a-4 b+$ $2 M+1$, and $8 E=a+2 M-1$.

\section{Generalized Cyclotomic Sequences of Order 4}

Throughout this section, let $p$ and $q$ be two distinct odd primes with $\operatorname{gcd}(p-1, q-1)=4$. Define $n=p q$ and $e=(p-1)(q-1) / 4$. Let $l$ be a prime and satisfy $\operatorname{gcd}(l, n)=1$.

The generalized cyclotomic sequence $\lambda^{\infty}$ of order 4 of period $n$ is defined by

$$
\lambda_{i}= \begin{cases}1, & \text { if } i \bmod n \in\{0\} \cup P \cup D_{0} \cup D_{1}, \\ 0, & \text { otherwise, }\end{cases}
$$

where $D_{0}$ and $D_{1}$ are defined by (3) and $P=p \mathbb{Z}_{q}^{*}$. Here, in this paper, we treat it as a sequence over a finite field $\mathbb{F}_{l}$, where $\operatorname{gcd}(l, n)=1$.

Denote $\operatorname{ord}_{n}(l)$ the multiplicative order of $l$ modulo $n$. Let $\zeta_{n}$ be an $n$-th primitive root of unity over $\mathbb{F}_{l^{\text {ord }} n}(l)$. For the sequence $\lambda^{\infty}$ defined by (15), we know

$$
\begin{aligned}
& \Lambda(x)=\sum_{i=0}^{n-1} \lambda_{i} x^{i}=1+\sum_{i \in P} x^{i}+\sum_{i \in D_{0}} x^{i}+\sum_{i \in D_{1}} x^{i}, \\
& \Lambda(1)=1+\frac{(p+1)(q-1)}{2} \bmod l .
\end{aligned}
$$

Define $\delta$ as follows:

$$
\delta= \begin{cases}1, & \text { if } l \mid 1+\frac{(p+1)(q-1)}{2}, \\ 0, & \text { otherwise. }\end{cases}
$$

Note that the generalized cyclotomic classes of order 2 are given by

$$
\begin{aligned}
& C_{0}=D_{0} \cup D_{2}, \\
& C_{1}=D_{1} \cup D_{3} .
\end{aligned}
$$

Define $\eta_{0}=\sum_{i \in C_{0}} \zeta_{n}^{i}$. The following lemma has been proven in [9].

Lemma 2 (see [9], Lemma 3.13). If $n \equiv 1(\bmod 4)$, then we have

$$
\eta_{0}\left(1-\eta_{0}\right)=-\frac{n-1}{4} .
$$

Hence, $\eta_{0} \in\{0,1\}$ if and only if $(n-1) / 4 \equiv 0(\bmod l)$.

To compute the linear complexity of $\lambda^{\infty}$, we need to compute $\operatorname{gcd}\left(x^{n}-1, \Lambda(x)\right)$. For this purpose, we require a number of auxiliary results.
TABLe 1: $p \not \equiv q(\bmod 8)$.

\begin{tabular}{lllll}
\hline$(i, j)$ & 0 & 1 & 2 & 3 \\
\hline 0 & $\mathrm{~A}$ & $\mathrm{~B}$ & $\mathrm{C}$ & $\mathrm{D}$ \\
1 & $\mathrm{E}$ & $\mathrm{E}$ & $\mathrm{D}$ & $\mathrm{B}$ \\
2 & $\mathrm{~A}$ & $\mathrm{E}$ & $\mathrm{A}$ & $\mathrm{E}$ \\
3 & $\mathrm{E}$ & $\mathrm{D}$ & $\mathrm{B}$ & $\mathrm{E}$ \\
\hline
\end{tabular}

TABle 2: $p \equiv q(\bmod 8)$.

\begin{tabular}{lllll}
\hline$(i, j)$ & 0 & 1 & 2 & 3 \\
\hline 0 & $\mathrm{~A}$ & $\mathrm{~B}$ & $\mathrm{C}$ & $\mathrm{D}$ \\
1 & $\mathrm{~B}$ & $\mathrm{D}$ & $\mathrm{E}$ & $\mathrm{E}$ \\
2 & $\mathrm{C}$ & $\mathrm{E}$ & $\mathrm{C}$ & $\mathrm{E}$ \\
3 & $\mathrm{D}$ & $\mathrm{E}$ & $\mathrm{E}$ & $\mathrm{B}$ \\
\hline
\end{tabular}

Lemma 3 (see [16], Lemma 5). Let $m$ be the least common multiple of two positive integers $m_{1}$ and $m_{2}$. The system of congruences

$$
\begin{aligned}
& x \equiv a_{1}\left(\bmod m_{1}\right), \\
& x \equiv a_{2}\left(\bmod m_{2}\right),
\end{aligned}
$$

has solutions if and only if

$$
\operatorname{gcd}\left(m_{1}, m_{2}\right) \mid a_{1}-a_{2},
$$

where $a \mid b$ means that a divides $b$. When condition (22) holds, the system of the congruences of (21) has only one solution modulo $m$.

Lemma 4. For $a \in D_{j}$, then $a D_{i}=D_{(i+j) \bmod 4}$.

Proof. To prove this lemma, we need to prove $x^{4} \in D_{0}$ for the integer $x$ defined by (1).

By the generalized Chinese reminder theorem, there exists an integer $s$ with $0 \leq s \leq e-1$ such that

$$
\left\{\begin{array}{l}
s \equiv 4(\bmod (p-1)) \\
s \equiv 0(\bmod (q-1))
\end{array}\right.
$$

That is, the integer $s$ satisfies

$$
\left\{\begin{array}{l}
x^{4} \equiv g^{s}(\bmod p) \\
x^{4} \equiv g^{s}(\bmod q) .
\end{array}\right.
$$

This $s$ is unique. Hence, $x^{4} \equiv g^{s}(\bmod p q)$ and $x^{4} \in D_{0}$. Because $\zeta_{n}$ is an $n$-th primitive root of unity over $\mathbb{F}_{l^{\text {ord }}(l)}$, we have

$$
\begin{aligned}
& \sum_{i \in P} \zeta_{n}^{i}=-1, \\
& \sum_{i \in Q} \zeta_{n}^{i}=-1 .
\end{aligned}
$$

By the definition of $\zeta_{n}$, we have

$$
\begin{aligned}
\zeta_{n}^{n}-1 & =\left(\zeta_{n}-1\right)\left(1+\sum_{i \in P} \zeta_{n}^{i}+\sum_{i \in Q} \zeta_{n}^{i}+\sum_{j=0}^{3} \sum_{i \in D_{j}} \zeta_{n}^{i}\right) \\
& =0 .
\end{aligned}
$$


Together with (25), we obtain

$$
\sum_{j=0}^{3} \sum_{i \in D_{j}} \zeta_{n}^{i}=1 .
$$

Define $t(x)=\sum_{i \in D_{1}} x^{i}+\sum_{i \in D_{2}} x^{i}$

Lemma 5. Let symbols be the same as before. Then,

$$
\Lambda\left(\zeta_{n}^{a}\right)= \begin{cases}\Lambda\left(\zeta_{n}\right), & a \in D_{0}, \\ t\left(\zeta_{n}\right), & a \in D_{1}, \\ -\left(\Lambda\left(\zeta_{n}\right)-1\right), & a \in D_{2}, \\ -\left(t\left(\zeta_{n}\right)-1\right), & a \in D_{3}, \\ -\frac{p-1}{2}, & a \in P, \\ \frac{q+1}{2}, & a \in Q\end{cases}
$$

Proof. By Lemma 4, $a D_{0}=D_{0}$ and $a D_{1}=D_{1}$ if $a \in D_{0}$. If $a \in D_{0}$, then $a P=P$ since $\operatorname{gcd}(a, q)=1$. Hence, we obtain

$$
\begin{aligned}
\Lambda\left(\zeta_{n}^{a}\right) & =1+\sum_{i \in P} \zeta_{n}^{a i}+\sum_{i \in D_{0} \cup D_{1}} \zeta_{n}^{a i} \\
& =1+\sum_{i \in P} \zeta_{n}^{i}+\sum_{i \in D_{0} \cup D_{1}} \zeta_{n}^{i} \\
& =\Lambda\left(\zeta_{n}\right) .
\end{aligned}
$$

If $a \in D_{1}$, by Lemma $4, a D_{0}=D_{1}$ and $a D_{1}=D_{2}$. By (25), we have

$$
\begin{aligned}
\Lambda\left(\zeta_{n}^{a}\right) & =1+\sum_{i \in P} \zeta_{n}^{a i}+\sum_{i \in D_{0} \cup D_{1}} \zeta_{n}^{a i} \\
& =\sum_{i \in D_{1}} \zeta_{n}^{i}+\sum_{D_{2}} \zeta_{n}^{i} \\
& =t\left(\zeta_{n}\right) .
\end{aligned}
$$

If $a \in D_{2}$, by Lemma $4, a D_{0}=D_{2}$ and $a D_{1}=D_{3}$. By (25) and (27), we obtain

$$
\begin{aligned}
\Lambda\left(\zeta_{n}^{a}\right) & =1+\sum_{i \in P} \zeta_{n}^{a i}+\sum_{i \in D_{0} \cup D_{1}} \zeta_{n}^{a i} \\
& =\sum_{i \in D_{2}} \zeta_{n}^{i}+\sum_{i \in D_{3}} \zeta_{n}^{i} \\
& =1-\sum_{i \in D_{0} \cup D_{1}} \zeta_{n}^{i} \\
& =1-\Lambda\left(\zeta_{n}\right) .
\end{aligned}
$$

If $a \in D_{3}$, by Lemma $4, a D_{0}=D_{3}$ and $a D_{1}=D_{0}$. By (27), we have

$$
\begin{aligned}
\Lambda\left(\zeta_{n}^{a}\right) & =1+\sum_{i \in P} \zeta_{n}^{a i}+\sum_{i \in D_{0} \cup D_{1}} \zeta_{n}^{a i} \\
& =\sum_{i \in D_{3}} \zeta_{n}^{i}+\sum_{D_{0}} \zeta_{n}^{i} \\
& =1-t\left(\zeta_{n}\right) .
\end{aligned}
$$

If $a \in P$, then $a P=P$. Then, by (25), we know

$$
\begin{aligned}
\Lambda\left(\zeta_{n}^{a}\right) & =1+\sum_{i \in P} \zeta_{n}^{a i}+\sum_{i \in\left(D_{0} \cup D_{1}\right)} \zeta_{n}^{a i} \\
& =\sum_{i \in\left(D_{0} \cup D_{1}\right)} \zeta_{n}^{a i} .
\end{aligned}
$$

When $s$ ranges over $\{0,1, \ldots, e-1\}$, it can be checked that $\left(D_{0} \cup D_{1}\right) \bmod q$ takes on each element of $\{1,2, \ldots, q-1\}$ exactly $(p-1) / 2$ times. It follows from (25) that

$$
\begin{aligned}
\sum_{i \in\left(D_{0} \cup D_{1}\right)} \zeta_{n}^{a i} & =\left(\frac{p-1}{2} \bmod l\right) \sum_{i \in P} \zeta_{n}^{i} \\
& =-\frac{p-1}{2} \bmod l .
\end{aligned}
$$

If $a \in Q$, then $a P=\{0\}$. Then,

$$
\begin{aligned}
\Lambda\left(\zeta_{n}^{a}\right) & =1+\sum_{i \in P} \zeta_{n}^{a i}+\sum_{i \in\left(D_{0} \cup D_{1}\right)} \zeta_{n}^{a i} \\
& =1+(q-1)+\sum_{i \in\left(D_{0} \cup D_{1}\right)} \zeta_{n}^{a i} .
\end{aligned}
$$

When $s$ ranges over $\{0,1, \ldots, e-1\},\left(D_{0} \cup D_{1}\right) \bmod p$ takes on each element of $\{1,2, \ldots, p-1\}$ exactly $(q-1) / 2$ times. It follows from (25) that

$$
\begin{aligned}
\Lambda\left(\zeta_{n}^{a}\right) & =1+(q-1)+\sum_{i \in\left(D_{0} \cup D_{1}\right)} \zeta_{n}^{a i} \\
& =1+(q-1)+\frac{q-1}{2} \sum_{i \in Q} \zeta_{n}^{i} \\
& =\frac{q+1}{2} \bmod l .
\end{aligned}
$$

Lemma 6 (see [20], Lemma 3.3). Let notations be the same as before. Then,
(1) $-1 \in D_{0}$ if and only if $p \neq \equiv(\bmod 8)$
(2) $-1 \in D_{2}$ if and only if $p \equiv q(\bmod 8)$ 
Lemma 7 (see [21], Lemmas 2 and 4). For each $w \in P \cup Q$,

$$
\left|\left\{D_{i} \cap\left(D_{j}+w\right)\right\}\right|= \begin{cases}\frac{(p-1)(q-1)}{16}, & \text { if } i \neq j, \\ \frac{(p-1)(q-5)}{16}, & \text { if } i=j \text { and } p \mid w \\ \frac{(p-5)(q-1)}{16}, & \text { if } \quad i=\text { and } q \mid w .\end{cases}
$$

Define

$$
\begin{aligned}
& \Delta_{1}=\frac{p-1}{2} \bmod l, \\
& \Delta_{2}=\frac{q+1}{2} \bmod l .
\end{aligned}
$$

Theorem 1. Let $(n-1 / 4) \equiv 0(\bmod l)$. Then, the linear complexity of the sequence $\lambda^{\infty}$ defined by (15) is given as follows:

(1) When $p \equiv q(\bmod 8)$ and $b / 2 \equiv 0(\bmod l)$, we have

$$
L_{l}(\lambda)=\left\{\begin{array}{lll}
\frac{p q+p+q-1}{2}-\delta, & \text { if } \quad \Delta_{1} \neq 0, \Delta_{2} \neq 0, \\
\frac{p q-p+q+1}{2}-\delta, & \text { if } & \Delta_{1} \neq 0, \Delta_{2}=0, \\
\frac{p q+p-q+1}{2}-\delta, & \text { if } & \Delta_{1}=0, \Delta_{2} \neq 0, \\
\frac{p q-p-q+1}{2}, & \text { if } & \Delta_{1}=0, \Delta_{2}=0 .
\end{array}\right.
$$

(2) When $q \neq \equiv(\bmod 8)$ and $\left(a^{2}+3\right) / 4 \equiv 0(\bmod l)$, we have

$$
L_{l}(\lambda)=\left\{\begin{array}{lll}
\frac{3 p q+p+q-1}{4}-\delta, & \text { if } & \Delta_{1} \neq 0, \Delta_{2} \neq 0, \\
\frac{3 p q-3 p+q+3}{4}-\delta, & \text { if } & \Delta_{1} \neq 0, \Delta_{2}=0, \\
\frac{3 p q+p-3 q+3}{4}-\delta, & \text { if } & \Delta_{1}=0, \Delta_{2} \neq 0, \\
\frac{3 p q-3 p-3 q+5}{4}, & \text { if } & \Delta_{1}=0, \Delta_{2}=0 .
\end{array}\right.
$$

(3) When $p \equiv q(\bmod 8)$ and $b / 2 \not \equiv 0(\bmod l)$ or $q \neq \equiv$ $(\bmod 8)$ and $\left(a^{2}+3\right) / 4 \not \equiv 0(\bmod l)$, we have

$$
L_{l}(\lambda)= \begin{cases}n-\delta, & \text { if } \Delta_{1} \neq 0, \Delta_{2} \neq 0, \\ n+1-p-\delta, & \text { if } \Delta_{1} \neq 0, \Delta_{2}=0, \\ n+1-q-\delta, & \text { if } \Delta_{1}=0, \Delta_{2} \neq 0, \\ n+2-p-q, & \text { if } \Delta_{1}=0, \Delta_{2}=0,\end{cases}
$$

where $a$ and $b$ are the integers defined in (14) and $\delta$ is defined in (18).

Proof. By definition, we have

$$
\Lambda\left(\zeta_{n}\right)^{2}=\left(\sum_{i \in D_{0}, j \in D_{0}}+\sum_{i \in D_{0}, j \in D_{0}}+\sum_{i \in D_{0}, j \in D_{0}}+\sum_{i \in D_{0}, j \in D_{0}}\right) \zeta_{n}^{i+j} .
$$

We first prove the conclusions for the case that $q \equiv p(\bmod 8)$. In this case, by Lemma $6,-1 \in D_{0}$ and $b$ must be even. By Table 2, in Lemma 1 and Lemma 7, we have

$$
\begin{aligned}
\Lambda\left(\zeta_{n}\right)^{2}= & \left.\sum_{i \in D_{0}, j \in D_{0}}+\sum_{i \in D_{0}, j \in D_{1}}+\sum_{i \in D_{1}, j \in D_{0}}+\sum_{i \in D_{1}, j \in D_{1}}\right) \zeta_{n}^{i-j} \\
= & ((0,0)+(3,3)+(0,1)+(3,0)) \sum_{i \in D_{0}} \zeta_{n}^{i} \\
& +((01,0)+(0,3)+(1,1)+(0,0)) \sum_{i \in D_{1}} \zeta_{n}^{i} \\
& +((2,0)+(1,3)+(2,1)+(1,0)) \sum_{i \in D_{2}} \zeta_{n}^{i} \\
& +((3,0)+(2,3)+(3,1)+(2,0)) \sum_{i \in D_{3}} \zeta_{n}^{i} \\
& +2\left(\frac{(p-1)(q-1)}{16} \sum_{i \in P} \zeta_{n}^{i}\right)+2\left(\frac{(p-1)(q-1)}{16} \sum_{i \in P} \zeta_{n}^{i}\right) \\
& +2\left(\frac{(p-1)(q-5)}{16} \sum_{i \in P} \zeta_{n}^{i}\right)+2\left(\frac{(p-5)(q-1)}{16} \sum_{i \in Q} \zeta_{n}^{i}\right) \\
& +\left|D_{0}\right|+\left|D_{1}\right| \\
= & \Lambda\left(\zeta_{n}\right)+b \quad \sum_{i \in\left(D_{0} \cup D_{2}\right)}^{\zeta_{n}^{i}-\frac{b}{2}+M+\frac{p+q-2}{2} .}
\end{aligned}
$$

From $(n-1) / 4 \equiv 0(\bmod l)$, we know $M+(p+q-2) /$ $2 \equiv 0(\bmod l)$. Hence,

$$
\Lambda\left(\zeta_{n}\right)^{2}=\Lambda\left(\zeta_{n}\right)+b \sum_{i \in\left(D_{0} \cup D_{2}\right)} \zeta_{n}^{i}-\frac{b}{2}
$$


Whence,

$$
\Lambda\left(\zeta_{n}\right)\left(\Lambda\left(\zeta_{n}\right)-1\right)=\frac{b}{2}\left(2 \sum_{i \in\left(D_{0} \cup D_{2}\right)} \zeta_{n}^{i}-1\right) .
$$

Note that $(n-1) / 4 \equiv 0(\bmod l)$. By Lemma 2 , we have $\sum_{i \in\left(D_{0} \cup D_{2}\right)} \zeta_{n}^{i} \in\{1,0\}$. It follows that

$$
2 \sum_{i \in\left(D_{0} \cup D_{2}\right)} \zeta_{n}^{i}-1 \in\{1,-1\}
$$

Similarly, we have

$$
t\left(\zeta_{n}\right)\left(t\left(\zeta_{n}\right)-1\right)=-\frac{b}{2}\left(2 \sum_{i \in\left(D_{0} \cup D_{2}\right)} \zeta_{n}^{i}-1\right) .
$$

By Lemma 5, (45), and (47), we know when $b / 2 \equiv 0(\bmod l)$, there are exactly half of $a^{\prime} s$ with $a \in \mathbb{Z}_{n}^{*}$ such that $s\left(\zeta_{n}^{a}\right)=0$. When $b / 2 \not \equiv 0(\bmod l), s\left(\zeta_{n}^{a}\right) \neq \equiv 0$ for all $a \in \mathbb{Z}_{n}^{*}$. Then, the desirable results on the linear complexity of the sequence $s$ follow from (11), (17), and Lemma 5 .

Now, we prove the conclusions for the case that $q \neq \equiv p(\bmod 8)$. In this case, by Lemma $6,-1 \in D_{2}$. It follows from Table 1 in Lemmas 1 and 7 that

$$
\begin{aligned}
\Lambda\left(\zeta_{n}\right)^{2}= & \left(\sum_{i \in D_{0}, j \in D_{2}}+\sum_{i \in D_{0}, j \in D_{3}}+\sum_{i \in D_{1}, j \in D_{2}}+\sum_{i \in D_{1}, j \in D_{3}}\right) \zeta_{n}^{i-j} \\
= & ((2,2)+(1,1)+(2,3)+(1,2)) \sum_{i \in D_{0}} \zeta_{n}^{i} \\
& +((3,2)+(2,1)+(3,3)+(2,2)) \sum_{i \in D_{1}} \zeta_{n}^{i} \\
& +((0,2)+(3,1)+(0,3)+(3,2)) \sum_{i \in D_{1}} \zeta_{n}^{i} \\
& +((1,2)+(0,1)+(1,3)+(0,2)) \sum_{i \in D_{1}} \zeta_{n}^{i} \\
& +4\left(\frac{(p-1)(q-1)}{16} \sum_{i \in P} \zeta_{n}^{i}\right)+4\left(\frac{(p-1)(q-1)}{16} \sum_{i \in Q}^{i} \zeta_{n}\right) \\
= & \Lambda\left(\zeta_{n}\right)+\frac{b\left(2 \sum_{i \in\left(D_{0} \cup D_{2}\right)} \zeta_{n}^{i}-1\right)-1}{2}+M-\frac{(p-1)(q-1)}{2}
\end{aligned}
$$

From $(n-1) / 4 \equiv 0(\bmod l)$, we have $M-((p-1)(q-$ $1)) / 2 \equiv 0(\bmod l)$. Hence,

$$
\Lambda\left(\zeta_{n}\right)^{2}=\Lambda\left(\zeta_{n}\right)+\frac{b\left(2 \sum_{i \in\left(D_{0} \cup D_{2}\right)} \zeta_{n}^{i}-1\right)-1}{2} .
$$

Whence,

$$
\Lambda\left(\zeta_{n}\right)\left(\Lambda\left(\zeta_{n}\right)-1\right)=\frac{b\left(2 \sum_{i \in\left(D_{0} \cup D_{2}\right)} \zeta_{n}^{i}-1\right)-1}{2} .
$$

Similarly, we get

$$
t\left(\zeta_{n}\right)\left(t\left(\zeta_{n}\right)-1\right)=-\frac{b\left(2 \sum_{i \in\left(D_{0} \cup D_{2}\right)} \zeta_{n}^{i}-1\right)+1}{2} .
$$

Since $\operatorname{gcd}(p-1, q-1)=4$ and $p \equiv q+4(\bmod 8)$, we get $p \equiv 1$ or $5(\bmod 8)$. Hence, $n=p q \equiv 5(\bmod 8)$. Together with $(n-1) / 4 \equiv 0(\bmod l)$, we obtain $l$ which is an odd prime. By the representation $n=a^{2}+4 b^{2}$ with $a \equiv 1(\bmod 4)$, we have

$$
\frac{n-1}{4}=\frac{a^{2}+3}{4}+(|b|-1)(|b|+1) \text {. }
$$

Therefore, $\left(a^{2}+3\right) / 4 \equiv 0(\bmod l)$ if and only if $(|b|-1)(|b|+1) \equiv 0(\bmod l)$. Since $l$ is odd, we obtain $(|b|-$ $1)(|b|+1) \equiv 0(\bmod l)$ if and only if $l$ divides one of $|b|-1$ and $|b|+1$. By Lemma 5 , if $\left(a^{2}+3\right) / 4 \equiv 0(\bmod l)$, there are exactly $(p-1)(q-1) / 4$ a's such that $s\left(\zeta_{n}^{a}\right)=0$ for $a \in \mathbb{Z}_{n}^{*}$. If $\left(a^{2}+3\right) / 4 \equiv 0(\bmod l), s\left(\zeta_{n}^{a}\right) \not \equiv 0$ for all $a \in \mathbb{Z}_{n}^{*}$. Then, the desirable conclusions on the linear complexity of the sequence $\lambda^{\infty}$ follow from (11), (17), (46), and Lemma 5.

For the case $l \notin D_{0}$, the linear complexity of the sequence $\lambda^{\infty}$ defined by (15) can also be determined in the following theorem.

Theorem 2. If $l \notin D_{0}$, then for the sequence $\lambda^{\infty}$ defined in (15), we have

$$
L_{l}(\lambda)= \begin{cases}n-\delta, & \text { if } \Delta_{1} \not \equiv 0, \Delta_{2} \not \equiv 0, \\ n+1-p-\delta, & \text { if } \Delta_{1} \not \equiv 0, \Delta_{2}=0, \\ n+1-q-\delta, & \text { if } \Delta_{1}=0, \Delta_{2} \neq 0, \\ n+2-p-q, & \text { if } \Delta_{1}=0, \Delta_{2}=0 .\end{cases}
$$

Proof. If $l \notin D_{0}$, then there exists $i \in\{1,2,3\}$ such that $i \in D_{i}$. No matter what $i$ is, there exists $j \in\{1,2,3\}$ satisfying the congruence equation $i j \equiv 2(\bmod 4)$. Then, $l^{j} \in D_{2}$ and

$$
\Lambda^{l j}\left(\zeta_{n}\right)=\Lambda\left(\zeta_{n}^{l^{j}}\right)=1-\Lambda\left(\zeta_{n}\right)
$$

This implies $\Lambda\left(\zeta_{n}\right) \notin\{0,1\}$.

Similarly, $t^{j^{j}}\left(\zeta_{n}\right)=t\left(\zeta_{n}^{l^{j}}\right)=1-t\left(\zeta_{n}\right)$ and $t\left(\zeta_{n}\right) \notin\{0,1\}$. Hence, $s\left(\zeta_{n}\right) \neq \equiv$ for all $a \in \mathbb{Z}_{n}^{*}$. Combining (11) and Lemma 5 , we have the desirable results.

For $j \in\{0,1,2,3\}$, define

$$
d_{j}(x)=\prod_{i \in D_{j}}\left(x-\zeta_{n}^{i}\right) .
$$

If $l \in D_{0}$, it can be easily proved that $d_{j}(x) \in \mathbb{F}_{l}[x]$ for all $j$. Let $d(x)=\prod_{j=0}^{3} d_{j}(x)$, then $d(x) \in \mathbb{F}_{l}[x]$. We have

$$
x^{n}-1=\prod_{i=0}^{n-1}\left(x-\zeta_{n}^{i}\right)=\frac{\left(x^{q}-1\right)\left(x^{p}-1\right) d(x)}{x-1} .
$$

Lemma 8 (see [20], Lemma 3.3). Let notations be the same as before. Then,

(1) $2 \in D_{0} \cup D_{2}$ if and only if $p \equiv q(\bmod 8)$

(2) $2 \in D_{1} \cup D_{3}$ if and only if $p \equiv q(\bmod 8)$ 
Lemma 9 (see [20], Lemma 3.5). Let $p \equiv q(\bmod 8)$. Then, there are exactly two representations over the integer ring $\mathbb{Z}$ :

$$
\begin{aligned}
p q & =a^{2}+4 b^{2}=a^{\prime 2}+4 b^{\prime 2}, \\
a & \equiv a^{\prime} \equiv 1(\bmod 4),
\end{aligned}
$$

where one of $b$ and $b^{\prime}$ is divided by 4 and another is exactly divided by 2 .

Lemma 10 (see [20], Corollary 3.9). Let $p \equiv q(\bmod 8)$. Fix $a$ common primitive root $g$ of $p$ and $q$. Then, $2 \in D_{0}$ if and only if the generalized cyclotomic numbers in Lemma 1 depend on the decomposition $n=a^{2}+4 b^{2}$ with $4 \mid b ; 2 \in D_{2}$ if and only if the generalized cyclotomic numbers depend on the decomposition $n=a^{2}+4 b^{2}$ with $2 \| b$, where $2 \| b$ means that $2 \mid b$ and $2^{2}+b$.

After the preparations above, we are ready to compute the linear complexity and minimal polynomials of the sequence defined in (15) over $\mathbb{F}_{2}$.

Corollary 1. Let $l=2$ and $n=a^{2}+4 b^{2} \equiv 1(\bmod 8)$. Then, $p \equiv q(\bmod 8)$ and the linear complexity and minimal polynomials of the sequence $\lambda^{\infty}$ defined by (15) are given as follows:

(1) When $4 \mid b$, then

$$
\begin{aligned}
& L_{2}(\lambda)= \frac{p q-q+p+1}{2}, \\
& m(x)= \begin{cases}\frac{\left(x^{n}-1\right)(x-1)}{\left(x^{q}-1\right) d_{2}(x) d_{1}(x)}, & \text { if } \Lambda\left(\zeta_{n}\right)=1 \text { and } t\left(\zeta_{n}\right)=0, \\
\frac{\left(x^{n}-1\right)(x-1)}{\left(x^{q}-1\right) d_{0}(x) d_{3}(x)}, & \text { if } \Lambda\left(\zeta_{n}\right)=0 \text { and } t\left(\zeta_{n}\right)=1, \\
\frac{\left(x^{n}-1\right)(x-1)}{\left(x^{q}-1\right) d_{0}(x) d_{1}(x)}, & \text { if } \Lambda\left(\zeta_{n}\right)=0 \text { and } t\left(\zeta_{n}\right)=0, \\
\frac{\left(x^{n}-1\right)(x-1)}{\left(x^{q}-1\right) d_{2}(x) d_{3}(x)}, & \text { if } \Lambda\left(\zeta_{n}\right)=1 \text { and } t\left(\zeta_{n}\right)=1 .\end{cases}
\end{aligned}
$$

(2) When $2 \| b$, then

$$
\begin{aligned}
& L_{2}(\lambda)=n+1-q, \\
& m(x)=\frac{\left(x^{n}-1\right)(x-1)}{x^{q}-1} .
\end{aligned}
$$

Proof. If $p \equiv q(\bmod 8)$, then $p \equiv q+4(\bmod 8)$ and $n=p q \equiv 5(\bmod 8)$. This contradicts with $n \equiv 1(\bmod 8)$. Hence, $p \equiv q(\bmod 8)$. Also, in this case, by Lemma 8 , $2 \in D_{0} \cup D_{2}$.

By (45), (47), and Lemma 10 , if $4 \mid b$, i.e., $2 \in D_{0}$, we have

$$
\begin{aligned}
\Lambda\left(\zeta_{n}\right) & \in\{0,1\}, \\
t\left(\zeta_{n}\right) & \in\{0,1\},
\end{aligned}
$$

and if $2 \| b$, i.e., $2 \in D_{2}$, we obtain

$$
\begin{aligned}
\Lambda\left(\zeta_{n}\right) & \notin\{0,1\}, \\
t\left(\zeta_{n}\right) & \notin 0,1\} .
\end{aligned}
$$

Then, the desired conclusions on the linear complexity and the minimal polynomial of the sequence $\lambda^{\infty}$ follow from (11) and Lemma 5.
If $p \not \equiv q(\bmod 8)$, then $n=p q \equiv 5(\bmod 8)$, and by Lemma $8,2 \in D_{1} \cup D_{3}$. Hence, by Theorem 2 , we have the following corollary.

Corollary 2. Let $l=2$ and $n \equiv 5(\bmod 8)$. Then, for the sequence $\lambda^{\infty}$ defined by (15), we have

$$
\begin{aligned}
& L_{2}(\lambda)=n+1-q, \\
& m(x)=\frac{\left(x^{n}-1\right)(x-1)}{x^{q}-1} .
\end{aligned}
$$

Example 1. Let $(p, q)=(5,13)$ and $\left(g_{1}, g_{2}\right)=(2,2)$. Then, $a=1$ and $b=4$. If $l=2$, Magma program shows that $2 \in D_{0}$ and $L_{2}(\lambda)=29$. If $l=3$, then $b / 2 \neq \equiv \bmod l$ and $L_{3}(\lambda)=65$.

Example 2. Let $(p, q)=(5,17)$ and $\left(g_{1}, g_{2}\right)=(2,3)$. Then, $a=-7$ and $b=3$. If $l=2$, it can be easily checked that $2 \in D_{3}$ and $L_{2}(\lambda)=69$. If $l=7$, then $\left(a^{2}+3\right) / 4 \neq 0 \bmod l$ and $L_{7}(\lambda)=85$.

\section{Data Availability}

No data were used to support this study. 


\section{Conflicts of Interest}

The authors declare that they have no conflicts of interest.

\section{Acknowledgments}

Q. Wang was supported by the National Science Foundation of China under grant no. 61602342, Natural Science Foundation of Tianjin under grant no. 18JCQNJC70300, the Science \& Technology Development Fund of Tianjin Education Commission for Higher Education under grant no. 2018KJ215, the Key Laboratory of Applied Mathematics of Fujian Province University (Putian University) under grant nos. SX201804 and SX201904, the China Scholarship Council (no. 201809345010), and NSFC (nos. 61972456, 61802281, and 2017KJ237). Y. Sun was supported by the National Natural Science Foundation of China (nos.61902429 and 11775306), Shandong Provincial Natural Science Foundation of China (nos. ZR2017MA001 and ZR2019MF070), Fundamental Research Funds for the Central Universities (nos. 19CX02058A and.17CX02030A), the Open Research Fund from Shandong Provincial Key Laboratory of Computer Networks under grant no. SDKLCN-2018-02, Key Laboratory of Applied Mathematics of Fujian Province University (Putian University) (nos. SX201702 and. SX201806), the Projects of International Cooperation and Exchanges NSFC-RFBR (no. 61911530130), and International Cooperation Exchange Fund of China University of Petroleum (no. UPCIEF2019020).

\section{References}

[1] C. Ding, "Pattern distributions of Legendre sequences," IEEE Transactions on Information Theory, vol. 44, no. 4, pp. 1693-1698, 1998.

[2] C. Ding, "Cyclic codes from cyclotomic sequences of order four," Finite Fields and Their Applications, vol. 23, pp. 8-34, 2013.

[3] C. Ding and T. Helleseth, "On cyclotomic generator of order r," Information Processing Letters, vol. 66, no. 1, pp. 21-25, 1998.

[4] C. Ding, T. Helleseth, and W. Shan, "On the linear complexity of Legendre sequences," IEEE Transactions on Information Theory, vol. 44, no. 3, pp. 1276-1278, 1998.

[5] V. A. Edemskii, "On the linear complexity of binary sequences on the basis of biquadratic and sextic residue classes," Discrete Mathematics and Applications, vol. 20, no. 1, pp. 75-84, 2010.

[6] V. Edemskiy, "About computation of the linear complexity of generalized cyclotomic sequences with period $p^{\mathrm{n}+1}$, , Designs, Codes and Cryptography, vol. 61, no. 3, pp. 251-260, 2011.

[7] V. Edemskiy and N. Sokolovskiy, "On the linear complexity of Hall's sextic residue sequences over $\backslash(\{\mathrm{GF}\}(\mathrm{q}) \backslash)$," Journal of Applied Mathematics and Computing, vol. 54, no. 1-2, pp. 297-305, 2017.

[8] J. H. Kim and H. Y. Song, "On the linear complexity of Hall's sextic residue sequences," IEEE Transactions on Information Theory, vol. 45, pp. 2094-2096, 2001.

[9] C. Ding, "Cyclic codes from the two-prime sequences," IEEE Transactions on Information Theory, vol. 58, no. 6, pp. 38813891, 2012.

[10] W. Meidl, "Remarks on a cyclotomic sequence," Designs, Codes and Cryptography, vol. 51, no. 1, pp. 33-43, 2009.
[11] Q. Wang, Y. Jiang, and D. Lin, "Linear complexity of binary generalized cyclotomic sequences over $\mathrm{GF}(q)$," Journal of Complexity, vol. 31, no. 5, pp. 731-740, 2015.

[12] Q. Wang, Y. Jiang, and D. Lin, "Linear complexity of DingHelleseth sequences of order 2 over GF(l)," Cryptography and Communications, vol. 8, no. 1, pp. 33-49, 2016.

[13] Q. Wang, Y. Jiang, D. Lin, and X. Guang, "On the linear complexity of new modified Jacobi sequences," IEICE Transactions on Fundamentals of Electronics, Communications and Computer Sciences, vol. E97-A, no. 11, pp. 22632266, 2014.

[14] E. Bai, X. Liu, and G. Xiao, "Linear complexity of new generalized cyclotomic sequences of order two of length $p q$," IEEE Transactions on Information Theory, vol. 51, no. 5, pp. 1849-1853, 2005.

[15] N. Brandstatter and A. Winterhof, "Some notes on the twoprime generator of order 2," IEEE Transactions on Information Theory, vol. 51, no. 10, pp. 3654-3657, 2005.

[16] C. Ding, "Linear complexity of generalized cyclotomic binary sequences of order 2," Finite Fields and Their Applications, vol. 3, no. 2, pp. 159-174, 1997.

[17] C. Ding, "Autocorrelation values of generalized cyclotomic sequences of order two," IEEE Transactions on Information Theory, vol. 44, no. 4, pp. 1699-1702, 1998.

[18] C. Ding and T. Helleseth, "New generalized cyclotomy and its applications," Finite Fields and Their Applications, vol. 4, no. 2, pp. 140-166, 1998.

[19] C. Fan and G. Ge, “A unified approach to Whiteman's and Ding-Helleseth's generalized cyclotomy over residue class rings," IEEE Transactions on Information Theory, vol. 60, no. 2, pp. 1326-1336, 2014.

[20] L. Hu, Q. Yue, and M. Wang, "The linear complexity of Whiteman's generalized cyclotomic sequences of period $p^{m+1} \mathrm{q}^{n+1}$," IEEE Transactions on Information Theory, vol. 58, no. 8, pp. 5534-5543, 2012.

[21] A. L. Whiteman, "A family of difference sets," Illinois Journal of Mathematics, vol. 6, no. 1, pp. 107-121, 1962.

[22] T. W. Cusick, C. Ding, and A. Renvall, Stream Ciphers and Number Theory, North Holland, Amsterdam, Netherlands, 1998.

[23] R. Lidl and H. Niederreiter, Finite Fields, Cambridge University Press, New York, NY, USA, 1997.

[24] W. J. Leveque, Topics in Number Theory, Vol. 1, AddisonWesley, Reading, MA, USA, 1956. 